TIFR/TH/99-40

\title{
CONTEXTUAL DETERMINISTIC QUANTUM MECHANICS
}

\author{
S.M. ROY \\ Tata Institute of Fundamental Research \\ Homi Bhabha Road, Mumbai 400 005, India \\ E-mail: shasanka@theory.tifr.res.in
}

\begin{abstract}
We present a simple proof of quantum contextuality for a spinless particle with a one dimensional configuration space. We then discuss how the maximally realistic deterministic quantum mechanics recently constructed by this author and V. Singh can be applied to different contexts.
\end{abstract}

PACS: 03.65 


\section{Context Dependence of Quantum Probabilities.}

A quantum state vector $|\psi\rangle$ specifies the probabilities $|\langle\alpha \mid \psi\rangle|^{2}=$ probability of observing the set of eigenvalues $\{\alpha\}=\left\{\alpha_{1}, \alpha_{2}, \cdots, \alpha_{n}\right\}$ of a complete commuting set of observables (CCS) $A=\left\{A_{1}, A_{2}, \cdots, A_{n}\right\}$ in the experimental situation or 'Context' where $A$ is observed. Equally, $|\psi\rangle$ specifies the probabilities $|\langle\beta \mid \psi\rangle|^{2}$ for observing the eigenvalues $\beta$ of a different CCS $B$ in the context where $B$ is measured. It is usually assumed that $A$ and $B$ cannot be measured simultaneously if they contain any mutually noncommuting observables, and hence that $|\langle\alpha \mid \psi\rangle|^{2}$ and $|\langle\beta \mid \psi\rangle|^{2}$ refer to different contexts.

Thus, quantum probabilities are inherently context dependent. Moreover, they cannot be embedded in context independent or "classical" probabilities. This is the essence of Gleason's theorem, Kochen-Specker theorem and Bell's theorem ${ }^{1}$, which apply to quantum systems with dimension of state vector space $\geq 3$.

An analogous question arises from a consideration of the Wigner distribution function ${ }^{2}$ $\rho(\vec{x}, \vec{p})$. It reproduces the correct context dependent quantum probability densities for position or momentum or for some components of position and commuting components of momentum as different marginals of the same (context independent) $\rho(\vec{x}, \vec{p})$. This is not a counter example to the contextuality theorem just quoted since the Wigner function is not positive definite and therefore cannot be given a probability interpretation. However, Cohen et $\mathrm{al}^{3}$ have constructed positive definite $\rho(\vec{x}, \vec{p})$ whose marginals reproduce the quantum position and momentum probability densities. In a series of investigations Roy and Singh $^{4}$ have gone much further; they constructed 'maximally realistic' causal quantum mechanics in which a single positive definite $\rho(\vec{x}, \vec{p})$ has marginals which reproduce not just the quantum position and momentum probability densities but also the quantum probability densities for $(n-1)$ other complete commuting sets of observables, where $n$ is the dimension of the configuration space. Further, for $n \geq 2$, Martin and Roy ${ }^{5}$ have proved a contextuality theorem: not all CCS can have their quantum probability densitites reproduced as marginals of a single positive definite phase space density. It remains a conjecture ${ }^{4}$ that $n+1$ is the maximum number of CCS whose probability densitites can be reproduced as marginals of a single positive definite $\rho(\vec{x}, \vec{p})$.

We shall prove here that a contextuality theorem analogous to the Martin-Roy theorem holds for $n=1$ too. We shall also give a simple proof of the Kochen-Specker theorem for a system of two spin half particles which is equivalent to an earlier proof of Peres and Mermin ${ }^{6}$.

We then discuss the Roy-Singh deterministic quantum mechanics for $n=1$ allowing simultaneous reality of position and momentum probability densities. Equally well, any other pair of canonically conjugate noncommuting variables can be simultaneously realized. However, the contextuality manifests itself: different choices of the simultaneously realised pair of dynamical variables lead to different phase space densities. The Roy-Singh deterministic quantum theory which allows $(n+1)$ CCS to be simultaneously realized is much more realistic than ordinary quantum mechanics or de Broglie-Bohm deterministic quantum mechanics ${ }^{7}$ (which allows only 1 CCS viz. position to be realized). It would be exciting to seek experimental evidence of simultaneous realization of $(n+1)$ CCS in one experimental setup. 


\section{A Simple Proof of Kochen-Specker theorem for a System of Two Spin Half Particles.}

Consider the possibility of a noncontextual microscopic theory ('hidden variable theory'?) more detailed than ordinary quantum mechanics in which a microstate assigns definite values for any set of observables even if the set contains noncommuting observables of quantum mechanics (which ordinarily refer to different contexts). Can such a theory be consistent with quantum predictions?

Quantum predictions imply that any commuting subset $A=\left\{A_{1}, A_{2}, \cdots, A_{n}\right\}$ of operators can be simultaneously measured. If the commuting operators obey the operator relation

$$
f\left(A_{1}, A_{2}, \cdots, A_{n}\right)=0
$$

the assigned values $v\left(A_{i}\right)$ in the noncontextual theory must also obey

$$
f\left(v\left(A_{1}\right), v\left(A_{2}\right), \cdots, v\left(A_{n}\right)\right)=0 .
$$

This is because the assigned set of values $\left\{v\left(A_{i}\right)\right\}$ must be an allowed set of simultaneous eigenvalues of $\left\{A_{1}, \cdots, A_{n}\right\}$; taking the expectation value of the operator equation (11) in the quantum state which has eigenvalues $v\left(A_{i}\right)$ for the operators $A_{i}$ we obtain Eq. (21). For a system of two particles of spin $1 / 2$ we shall repeatedly use special cases of Eqns. (11) and (2), viz. if $[A, B]=0$, then

$$
v(A B)=v(A) v(B), v(A+B)=v(A)+v(B) .
$$

Consider the operator relation involving the Pauli spin operators $\sigma_{1 x}, \sigma_{1 y}$ for particle 1 , and analogous operators for particle 2 ,

$$
\left(\left(\sigma_{1 x} \sigma_{2 y}\right)\left(\sigma_{1 y} \sigma_{2 x}\right)\right)+\left(\left(\sigma_{1 x} \sigma_{2 x}\right)\left(\sigma_{1 y} \sigma_{2 y}\right)\right)=0
$$

where each parenthesis encloses two commuting operators. Hence, applying (3) repeatedly we obtain

$$
2 v\left(\sigma_{1 x}\right) v\left(\sigma_{2 y}\right) v\left(\sigma_{1 y}\right) v\left(\sigma_{2 x}\right)=0 .
$$

But the left-hand side equals \pm 2 since the eigenvalue of a Pauli matrix equals \pm 1 . The contradiction shows the impossibility of a noncontextual extension of quantum mechanics, which is the Kochen-Specker theorem. This simple proof following immediately from Eq. (四) captures the essence of Mermin's earlier proof ${ }^{6}$.

It is worth pointing out that the contradiction follows from two hypotheses.

(i) Simultaneous assignment of values to the operators

$$
\sigma_{1 x} \sigma_{2 x}, \sigma_{1 x} \sigma_{2 y}, \sigma_{1 y} \sigma_{2 x}, \sigma_{1 y} \sigma_{2 y}, \sigma_{1 z} \sigma_{2 z}, \sigma_{1 x}, \sigma_{1 y}, \sigma_{2 x}, \sigma_{2 y}
$$

These assumptions are features also of Bell's theorem on violation of Einstein locality. It is not an additional assumption that the products $\left(\sigma_{1 x} \sigma_{2 y}\right)\left(\sigma_{1 y} \sigma_{2 x}\right),\left(\sigma_{1 x} \sigma_{2 x}\right)\left(\sigma_{1 y} \sigma_{2 y}\right)$ (which superficially involve factors not spacelike separated) have values, since these products are equal to $\sigma_{1 z} \sigma_{2 z}$ and $-\sigma_{1 z} \sigma_{2 z}$ respectively. 
(ii) The values of any commuting subset of the operators (6) must be an allowed set of their simultaneous eigenvalues.

In the cases of the subsets $\left\{\sigma_{1 x} \sigma_{2 y}, \sigma_{1 y} \sigma_{2 x}, \sigma_{1 z} \sigma_{2 z}\right\},\left\{\sigma_{1 x} \sigma_{2 x}, \sigma_{1 y} \sigma_{2 y}, \sigma_{1 z} \sigma_{2 z}\right\}$ for which commutation of the operators cannot be derived from a locality postulate, this KochenSpecker assumption is additional to Bell's assumptions.

3. Phase Space Contextuality Theorem For One Dimensional Configuration Space.

Martin and Roy $^{5}$ have shown that in configuration space dimension $\geq 2$, there cannot exist a positive definite phase space distribution function whose marginals coincide with the quantum position probability density, momentum probability density and joint probability densities of commuting components of position and momentum. I shall prove here an analogous contextuality theorem for configuration space dimension 1 .

Let $X_{o p}(\theta), P_{o p}(\theta)$ be a continuum of $\theta$-dependent operators defined by

$$
\left(\begin{array}{c}
X_{o p}(\theta) \\
P_{o p}(\theta) /(m \omega)
\end{array}\right)=\left(\begin{array}{cc}
\cos \theta & \sin \theta \\
-\sin \theta & \cos \theta
\end{array}\right)\left(\begin{array}{c}
x_{o p} \\
p_{o p} /(m \omega)
\end{array}\right)
$$

where $x_{o p}$ and $p_{o p}$ are the usual position and momentum operators. The constants $m, w$ have dimensions $[M],\left[T^{-1}\right]$ respectively. Then,

$$
\left[X_{o p}(\theta), P_{o p}(\theta)\right]=i \hbar
$$

We then prove the following contextuality theorem.

Theorem. There exist state vectors $|\psi\rangle$ for which it is impossible to find a positive definite phase space density $\rho(x, p)$ with the property

$$
\int_{X(\theta) \text { fixed }} \rho(x, p) d P(\theta)=|\langle X(\theta) \mid \psi\rangle|^{2} \text {, for all } \theta \text {. }
$$

Here $x, p, X(\theta), P(\theta)$ denote $c$-no. values belonging to the spectrum of the operators $x_{o p}, p_{o p}$, $X_{o p}(\theta), P_{o p}(\theta)$, and the integral over $P(\theta)$ is done for a fixed value of $X(\theta)$; i.e., we reexpress $\rho(x, p)$ in terms of new variables $X(\theta), P(\theta)$,

$$
\rho(x, p)=\rho_{\theta}(X(\theta), P(\theta))
$$

and then integrate over $P(\theta)$.

Proof. Consider the energy eigen function for the first excited state of the oscillator,

$$
\langle x \mid \psi\rangle=\frac{1}{\sqrt{2}}\left(\frac{m \omega}{\pi \hbar}\right)^{1 / 4} 2 \sqrt{\frac{m \omega}{\hbar}} x \exp \left(-\frac{1}{2} \frac{m \omega}{\hbar} x^{2}\right) .
$$

The $X(\theta)$ representative of the same state vector $|\psi\rangle$ is given by a simple calculation

$$
\langle X(\theta) \mid \psi\rangle=\int d x\langle X(\theta) \mid x\rangle\langle x \mid \psi\rangle
$$


where $\langle X(\theta) \mid x\rangle$ is found from the differential eqn.

$$
\begin{aligned}
X(\theta)\langle x \mid X(\theta)\rangle & =\left\langle x\left|\cos \theta x_{o p}+\frac{\sin \theta}{m \omega} p_{o p}\right| X(\theta)\right\rangle \\
& =x \cos \theta\langle x \mid X(\theta)\rangle-i \hbar \frac{\sin \theta}{m \omega} \frac{\partial}{\partial x}\langle x \mid X(\theta)\rangle .
\end{aligned}
$$

We thus find

$$
\begin{aligned}
\langle X(\theta) \mid \psi\rangle= & \left(\frac{m \omega}{\pi \hbar}\right)^{1 / 4} \sqrt{\frac{2 m \omega}{\hbar}} X(\theta) \exp \left[i\left(\frac{3 \theta}{2}-\frac{\pi}{4}\right)\right. \\
& \left.+\frac{m \omega}{\hbar}\left(\frac{i}{2} \cot \theta(X(\theta))^{2}-\frac{1}{2}(X(\theta))^{2}\right)\right] .
\end{aligned}
$$

The most important point to notice is that

$$
\langle X(\theta) \mid \psi\rangle=0 \text { at } X(\theta)=0
$$

If $\exists$ positive definite $\rho(x, p)$ obeying (9), then the equation

$$
\int_{X(\theta)=0} d P(\theta) \rho(x, p)=0
$$

implies that $\rho(x, p)$ must vanish identically on the line

$$
\cos \theta x+\sin \theta \frac{p}{m \omega}=0 .
$$

For any given $x, p$ we can find $\theta$ which solves this equation. Hence $\rho(x, p)$ must vanish identically for all $x, p$. This is a contradiction because Eq. (9) does not allow it. Hence a positive definite phase space density reproducing the quantum marginals $|\langle X(\theta) \mid \psi\rangle|^{2}$ for all $\theta$ cannot be found.

The method of proof is similar to that of Martin and Roy ${ }^{5}$ except for the fact that here I use canonical transformations which mix position and momentum.

\section{Contextual Deterministic Quantum Mechanics.}

De Broglie and Bohm ${ }^{7}$ (dBB) constructed a deterministic quantum mechanics in which only one CCS, namely position could have (objective) reality independent of observations since only position probability densities are correctly reproduced as a marginal of the $\mathrm{dBB}$ phase space density,

$$
\rho_{d B B}=|\psi(\vec{x}, t)|^{2} \delta\left(\vec{p}-\vec{p}_{d B B}(\vec{x}, t)\right) .
$$

The individual trajectories given by a causal Hamiltonian are

$$
\vec{p}_{d B B}=m \frac{d \vec{x}}{d t}=\vec{\nabla} S(\vec{x}, t),
$$


where $\psi=R \exp (i S / \hbar)$, with $R$ and $S$ real; the ensemble of trajectories ensure that at each time the quantum probability density $|\psi(\vec{x}, t)|^{2}$ is reproduced. It is easy to show that ${ }^{8}$

$$
\int \rho_{d B B} d \vec{x} \neq|\langle\vec{p} \mid \psi(t)\rangle|^{2}
$$

In $n$ dimensional configuration space Roy and Singh $^{4}$ constructed a new deterministic quantum mechanics in which the quantum probability densities of $n+1$ CCS are simultaneously reproduced as marginals of one positive definite phase space density. The individual trajectories are given by a causal Hamiltonian and hence the phase space density is constant along the trajectory (Liouville property). The new mechanics is conjectured to be maximally realistic in the sense that it might be impossible to reproduce more than $n+1$ CCS as marginals of a positive definite phase space density. For $n=1$, two simple phase space densities with this property given by Roy and Singh are

$$
\rho(x, p, t)=|\langle x \mid \psi\rangle|^{2}|\langle p \mid \psi\rangle|^{2} \delta\left(\int_{-\infty}^{p} d p^{\prime}\left|\left\langle p^{\prime} \mid \psi\right\rangle\right|^{2}-\int_{-\infty}^{\epsilon x} d x^{\prime}\left|\left\langle\epsilon x^{\prime} \mid \psi\right\rangle\right|^{2}\right)
$$

where $\epsilon= \pm 1$, and we have written $|\psi\rangle=|\psi(t)\rangle$ for brevity. It is easy to check from this expression which is symmetric between $x$ and $p$ that both $|\langle x \mid \psi\rangle|^{2}$ and $|\langle p \mid \psi\rangle|^{2}$ are reproduced as marginals.

Nevertheless the mechanics is not context independent. We already know from the proof of the previous section that we cannot reproduce $|\langle X(\theta) \mid \psi\rangle|^{2}$ for all $\theta$ as marginals. In spite of the fact that the operators $X_{o p}(\theta)$ are in general non-trivial linear combinations of the position and momentum variables which may be somewhat unfamiliar, they are self-adjoint operators and therefore observables. For any given $\theta$, the Roy-Singh procedure can be used to obtain a phase space density which reproduces $|\langle X(\theta) \mid \psi\rangle|^{2}$ and $|\langle P(\theta) \mid \psi\rangle|^{2}$ as marginals. (Just replace $x, p$ by $X(\theta), P(\theta)$ on the right-hand side of Eq. (15)). The important point is that these phase space densities are $\theta$-dependent and therefore must refer to different contexts: each context refers to realizing $|\langle X(\theta) \mid \psi\rangle|^{2}$ and $|\langle P(\theta) \mid \psi\rangle|^{2}$ for a definite $\theta$. Both the features of the Roy-Singh theory, (i) realizing two probability densities instead of one in $d B B$ theory, and (ii) the inevitable contextuality, are exhibited in the phase space density. We may mention that a $d B B$ like theory which reproduces only one marginal, but with position replaced by a non-trivial linear combination of position and momentum has been considered before in the context of a quantum potential approach to a class of quantum cosmological models ${ }^{9}$.

For $n>1$, it is even easier to exhibit the context dependence of the Roy-Singh phase space densities. E.g. for $n=2$, consider

$$
\begin{aligned}
\rho_{\theta}(\vec{x}, \vec{p}, t)= & \left|\left\langle x_{1}(\theta), x_{2}(\theta) \mid \psi\right\rangle\right|^{2}\left|\left\langle p_{1}(\theta), x_{2}(\theta) \mid \psi\right\rangle\right|^{2}\left|\left\langle p_{1}(\theta), p_{2}(\theta) \mid \psi\right\rangle\right|^{2} \\
& \delta\left(\int_{-\infty}^{p_{1}(\theta)}\left|\left\langle p_{1}^{\prime}, x_{2}(\theta) \mid \psi\right\rangle\right|^{2} d p_{1}^{\prime}-\int_{-\infty}^{x_{1}(\theta)}\left|\left\langle x_{1}^{\prime}, x_{2}(\theta) \mid \psi\right\rangle\right|^{2} d x_{1}^{\prime}\right) \\
& \delta\left(\int_{-\infty}^{p_{2}(\theta)}\left|\left\langle p_{1}(\theta), p_{2}^{\prime} \mid \psi\right\rangle\right|^{2} d p_{2}^{\prime}-\int_{-\infty}^{x_{2}(\theta)}\left|\left\langle p_{1}(\theta), x_{2}^{\prime} \mid \psi\right\rangle\right|^{2} d x_{2}^{\prime}\right),
\end{aligned}
$$


where

$$
\left(\begin{array}{l}
x_{1}(\theta) \\
x_{2}(\theta)
\end{array}\right)=\left(\begin{array}{cc}
\cos \theta & \sin \theta \\
-\sin \theta & \cos \theta
\end{array}\right)\left(\begin{array}{l}
x_{1} \\
x_{2}
\end{array}\right)
$$

and a similar equation defines $p_{1}(\theta), p_{2}(\theta)$ in terms of $p_{1}, p_{2}$. The phase space densities depend on $\theta$; for each $\theta$, the three indicated quantum probability densities $\left|\left\langle x_{1}(\theta), x_{2}(\theta) \mid \psi\right\rangle\right|^{2}$, $\left|\left\langle p_{1}(\theta), x_{2}(\theta) \mid \psi\right\rangle\right|^{2}$ and $\left|\left\langle p_{1}(\theta), p_{2}(\theta) \mid \psi\right\rangle\right|^{2}$ are reproduced as marginals. The causal Hamiltonians corresponding to Eqns. (15) and (16) have also been calculated.

The experimental realization of contexts in which some specific $(n+1)$ CCS are simultaneously realized remains a challenge.

Acknowledgements. I am grateful to Deepak Kumar and other organizers of the Conference on 'Quantum Many Body Physics' at the Jawaharlal Nehru University, New Delhi, March 5-7 for inviting me to present these results. It is a great pleasure to acknowledge that the wide physics interests of R. Rajaraman (in whose honour this meeting was held) has always been inspiring. Many of the ideas reported here have been developed in collaboration with Virendra Singh.

\section{$\underline{\text { References }}$}

1. A.M. Gleason, J. Math. \& Mech. ㅁ, 885 (1957); S. Kochen and E.P. Specker, ibid 17, 59 (1967); J.S. Bell, Physics 1, 195 (1964).

2. E. Wigner, Phys. Rev. 뚜, 749 (1932).

3. L. Cohen and Y.I. Zaparovanny, J. Math. Phys. 21, 794 (1980); L. Cohen, ibid $\underline{25}$, 2402 (1984).

4. S.M. Roy and V. Singh, Mod. Phys. Lett. A10, 709 (1995); S.M. Roy and V. Singh, Phys. Lett. A255, 201 (1999); S.M. Roy and V. Singh, in 'Deterministic Quantum Mechanics in One Dimension', p. 434, R. Cowsik (Ed.), Proceedings of International Conference on Non-accelerator Particle Physics, 2-9 January, 1994, Bangalore, World Scientific (1995); V. Singh, Pramana 49, 5 (1997); S.M. Roy, Pramana 포, 597 (1998).

5. A. Martin and S.M. Roy, Phys. Lett. B350, 66 (1995).

6. A. Peres, Phys. Lett. $\underline{\text { A151, }} 107$ (1990); N.D. Mermin, Phys. Rev. Lett. $\underline{65}, 3373$ (1990).

7. L. de Broglie, Non-linear Wave Mechanics, A Causal Interpretation, Elsevier, 1960; D. Bohm, Phys. Rev. 85, 166, 180 (1952); P.R. Holland, The Quantum Theory of Motion, Cambridge University Press 1993 and Foundations of Physics 28, 881 (1998); D. Bohm and B.J. Hiley, The Undivided Universe, Routledge, London, 1993; E. Deotto and G.C. Ghirardi, Foundations of Physics $\underline{28}$, 1 (1998).

8. T. Takabayasi, Prog. Theor. Phys. $\underline{8}, 143$ (1952).

9. A. Blaut and J. Kowalski-Glikman, Class. Quant. Grav. 13, 39 (1996). 